\title{
SIZE-DEPENDENT ELASTIC PROPERTIES OF Ni NANOFILMS BY MOLECULAR DYNAMICS SIMULATION
}

\author{
ZHENYU YANG and YA-PU ZHAO* \\ State Key Laboratory of Nonlinear Mechanics (LNM), Institute of Mechanics, \\ Chinese Academy of Sciences, Beijing 100080, People's Republic of China \\ *yzhao@imech.ac.cn
}

Received 19 December 2006

\begin{abstract}
Size-dependent elastic properties of Ni nanofilms are investigated by molecular dynamics (MD) simulations with embedded atom method (EAM). The surface effects are considered by calculating the surface relaxation, surface energy, and surface stress. The Young's modulus and yield stress are obtained as functions of thickness and crystallographic orientation. It is shown that the surface relaxation has important effects on the the elastic properties at nanoscale. When the surface relaxation is outward, the Young's modulus decreases with the film thickness decreasing, and vice versa. The results also show that the yield stresses of the films increase with the films becoming thinner. With the thickness of the nanofilms decreasing, the surface effects on the elastic properties become dominant.
\end{abstract}

Keywords: Molecular dynamics simulation; size-dependent elastic properties; nanofilms; surface relaxation; Young's modulus; yield stress.

\section{Introduction}

With the rapid development of nanoelectromechanical system (NEMS) technologies ${ }^{1}$ the information of mechanical properties of nanofilms has become indispensable for NEMS, where nanofilms are used as devices, sensors, and other structural materials. In nanostructures, surface to volume ratio is large and this difference is pivotally important. Elastic constants near surfaces are different from their values in the bulk of solids. ${ }^{2}$ For a metal surface, the atoms near the surface are exposed to different forces from those in the interior of the material. The atoms near the surface tend to relax mostly perpendicular to the surface to minimize the total energy.
Theoretical investigation of the size-dependent elastic constants of nanoscale single-crystal nanofilms has been carried out extensively. Guo and $Z_{\text {Zhao }}^{3}$ presented a three-dimensional model considering surface relaxation of the nanofilm by introducing a relaxation coefficient $k$ to modify the lattice constant of surface atoms. Their results show that the relaxation coefficient is the dominant factor for the size-dependent properties. Workum et al. ${ }^{4}$ presented a method for the calculation of the local elastic constants in inhomogeneous systems. In this method, the face-centered cubic (FCC) crystal is considered as Lennard--Jones ( $\mathrm{L}-\mathrm{J}$ ) particles and the local elastic constants of a nearest-neighbor L-J FCC crystal

*Corresponding author. 
in the bulk and in a thin film are obtained. Liang et al..$^{5}$ established a model for size-dependent elastic modulus of $\mathrm{Cu}$ and $\mathrm{Au}$ thin films based on the consideration of size-dependent atomic distance and bond energy. Their theoretical results show that the elastic modulus of metallic free thin films increases as the thickness of the films decreases.

Using a combination of molecular statics and $a b$ initio calculations, Zhou et $a l^{2}{ }^{2}$ show that a solid surface can be either softer or stiffer elastically than the corresponding bulk, which depends on the competition between the atomic coordination and electron redistribution on the surface. The surface-stress-induced phase transformation in gold nanowires is studied by atomistic simulation, ${ }^{6}$ which shows that the surface stress is an important factor of surface effects. Interface stresses and their effects on the elastic moduli of metallic multilayers are also calculated by atomic simulation. ${ }^{7}$ Wan et al. ${ }^{8}$ used modified EAM to obtain the multilayer relaxation for multiplicate FCC metals, which suggests when the surface stress is negative, the surface relaxation is inward; otherwise, the relaxation is outward. Several experiments have also demonstrated that the properties of nanometer scale materials can be quite different from those of bulk materials due to the effects of the surfaces. ${ }^{9,10}$

The sign and magnitude of the relaxation of the topmost atomic layers are mainly determined by the rearrangements of the surface state charge. ${ }^{11}$ However, the essence of surface effects on the sizedependent properties is not explicit enough. In this work, we employ the MD to try to give an insight into the surface relaxation and surface stress including their effects on the elastic properties of nanofilms.

\section{Method}

We perform MD simulations using $\mathrm{EAM}^{12}$ as the underlying atomic interaction model. Within the framework of the EAM, the total internal energy of the system of $N$ atoms is described as the energy required to embed these $N$ atoms into the homogeneous electron gas. The total energy $U$ for a system of atoms can be written as

$$
U=\sum_{i}^{N}\left[F_{i}\left(\bar{\rho}_{i}\right)+\frac{1}{2} \sum_{j \neq i}^{N} \phi_{i j}\left(r_{i j}\right)\right],
$$

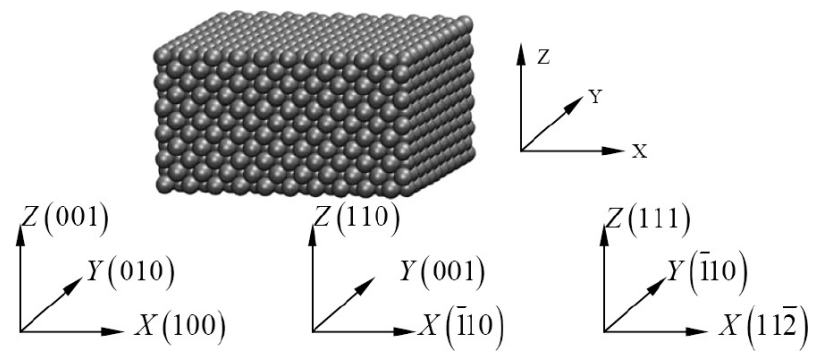

Fig. 1. Schematic of Ni nanofilm crystallographic orientations and transverse directions.

where $F_{i}\left(\bar{\rho}_{i}\right)$ is the embedding energy required to place atom $i$ in a local electron density $\bar{\rho}_{i}$, and $\phi_{i j}$ is the two-body potential between atoms $i$ and $j$, and $r_{i j}$ is the distance between atoms $i$ and $j$. The summation above is over the total number of atoms $N$ in the system.

Nanofilms with (100), (110) and (111) crystallographic orientations are created out of a bulk $\mathrm{Ni}$ FCC crystal (Fig. 1). The nanofilms are all 15 cubic lattice units long at the horizontal direction, with their thickness varying from about 1.06 to $3.52 \mathrm{~nm}$. In order to obtain equilibrium configurations, the nanofilms are relaxed to an equilibrium minimum energy configuration with periodical boundary conditions for the horizontal direction, and then thermally equilibrated to $300 \mathrm{~K}$ for $20 \mathrm{ps}$ using a Nosé-Hoover thermostat $^{13,14}$ with a time step of $1 \mathrm{fs}$. In the equilibrium process, a constant number of atoms, constant volume, and constant temperature (NVT) assembly are adopted. The thermostat is removed prior to loading, ensuring adiabatic loading conditions. The applied strain rates for each thin film are $3 \times 10^{7} \mathrm{~s}^{-1}$. The equations of motion are integrated in time using a velocity Verlet algorithm, and all MD simulations in this study are performed using the parallelized code LAMMPS. ${ }^{15,16}$

Moreover, the surface stresses of relaxed (100), (110), and (111) surfaces are calculated. It is assumed that the surface normal is defined as the $Z$-direction with the $X-Y$ plane lying in the surface (Fig. 1). The surface stress is a second-rank tensor. It can be expressed $a^{17}$ :

$$
\tau_{x y}=\frac{1}{A} \frac{\partial(\gamma A)}{\partial \epsilon_{x y}},
$$

where $A$ is the area of the surface, $\gamma$ the surface energy, and $\epsilon_{x y}$ the strain tensor. The surface stress tensor is isotopic on the (100) and (111) boundary 
planes. ${ }^{7}$ The average surface stress may then also be written as:

$$
\tau=\gamma+\frac{\partial \gamma}{\partial \epsilon}
$$

For the calculation of the Young's modulus from the stress versus strain curves, these stresses are calculated using the virial theorem, ${ }^{18}$ which takes the form

$\sigma=\frac{1}{\Omega}\left[-\sum_{i} m_{i} \mathbf{v}_{i} \otimes \mathbf{v}_{i}+\frac{1}{2} \sum_{i} \sum_{j} \mathbf{r}_{i j} \otimes \mathbf{f}_{i j}\right]$,

where the summation is over all the atoms occupying the total volume $\Omega$, and the first term is the kinetic energy component for atom $i, m_{i}$ for the mass of the atom $i$, and $\mathbf{v}_{i}$ for the velocity vector of atom $i . \mathbf{r}_{i j}=$ $\mathbf{r}_{i}-\mathbf{r}_{j}$ and $\mathbf{f}_{i j}$ is the interior atomic force applied on atom $i$ by atom $j$.

\section{Results and Discussion}

\subsection{Surface relaxation}

Slabs are used to simulate the surfaces. In order to investigate the surface relaxation, the top five layers of the thin films are set as the surface layers. The film models used to study the surface relaxation are made up of 15 layers, which are enough to simulate the surface characteristics. ${ }^{8}$ The computationally generated thin films are dynamically relaxed to obtain their equilibrium states at $300 \mathrm{~K}$ for $20 \mathrm{ps}$ with periodical boundary conditions applied along the horizontal direction.

The average distances between the layers are taken as the reference distances. For the bulk system, the distances between the layers along the (100), (110), and (111) orientation are $1.760 \AA, 1.246 \AA$, and $2.035 \AA$, respectively. The changes in these distances after relaxation are listed in Table 1.

Table 1. The surface relaxation of the top five layers of $\mathrm{Ni}$ nanofilms. The positive and negative values indicate the relaxation outward and inward, respectively.

\begin{tabular}{rcccc}
\hline Ni surface & $\Delta d_{12}$ & $\Delta d_{23}$ & $\Delta d_{34}$ & $\Delta d_{45}$ \\
\hline (100) This work & $0.90 \%$ & $0.95 \%$ & $0.13 \%$ & $0.05 \%$ \\
Exp. [19] & $\begin{array}{c}1.1 \pm 1.1 \% \\
-1.28 \%\end{array}$ & $-0.60 \%$ & $0.56 \%$ & $-0.11 \%$ \\
(110) This work & $-1.0 \pm 1.6 \%$ & & & \\
Exp. [19] & $-5.0 \pm 1.5 \%$ & $0.02 \%$ \\
(111) This work & $-1.39 \%$ & $0.59 \%$ & $-0.14 \%$ \\
Exp. [19] & $-1.2 \pm 1.2 \%$ & & & \\
\hline
\end{tabular}

For the $\mathrm{Ni}(100)$ surface, the experimental results showed a small outward relaxation of $1.1 \pm 1.1 \% .{ }^{19}$ The MD in this work gives an expansion of $0.9 \%$ which is consistent with the experimental results. For $\mathrm{Ni}(110)$ and $\mathrm{Ni}(111)$, the inward relaxation of the first layer was demonstrated by LEED, ${ }^{19}$ which is also shown in the MD results. But not all the layers have the same relaxation direction. The third layer of $\mathrm{Ni}(110)$, the second and the fourth layers of $\mathrm{Ni}(111)$ have the inverse relaxation directions. On the whole, the changes of the relaxation distances decrease from the top surface layer to the bulk.

\subsection{Surface energy and surface stress}

In order to get the surface energy, the nanofilms with periodical boundary conditions in all the directions are also equilibrated to minimal energy states. The surface energies of the $\mathrm{Ni}(100), \mathrm{Ni}(110)$, and $\mathrm{Ni}(111)$ nanofilms calculated by MD are listed in Table 2 .

It is shown that the surface energy of $\mathrm{Ni}(110)$ surface is the highest, while $\mathrm{Ni}(111)$ has the lowest surface energy. The surface energies calculated by MD with EAM agree well with the experimental data. Surface stress can be obtained as shown in Table 3 by Eq. (3). The surface stresses of the clean surfaces are all positive, which are also seen in the $a b$ initio calculations. $^{20}$

\subsection{Elastic modulus and yield stress}

After geometric construction, the naonfilms are first equilibrated at $300 \mathrm{~K}$ for $20 \mathrm{ps}$. Approximation to

Table 2. The surface energy of the $\mathrm{Ni}$ nanofilms in $\mathrm{J} / \mathrm{m}^{2}$.

\begin{tabular}{lccc}
\hline & $\gamma_{100}$ & $\gamma_{110}$ & $\gamma_{111}$ \\
\hline EAM & 1.585 & 1.732 & 1.447 \\
Exp. [17] & 1.532 & 1.647 & 1.431 \\
\hline
\end{tabular}

Table 3. Surface stress $\tau_{x x}$ of the relaxed $\mathrm{Ni}$ surfaces. All the stresses are in $\mathrm{N} / \mathrm{m}$.

\begin{tabular}{lccc}
\hline & 100 & 110 & 111 \\
\hline EAM & 1.697 & 2.809 & 1.648 \\
Ref. 8 & 1.270 & - & 0.430 \\
\hline
\end{tabular}


quasi-static tensile loading in each deformation increment is achieved in two steps. For the calculation of elastic modulus, strains in the range of $0 \%-2 \%$ are applied. This strain range is divided into 20 points. At each point, all atoms are allowed to relax to the minimum energy corresponding to the horizontal strains. The stresses in MD simulation are calculated by the virial theorem. The Young's modulus of Ni nanofilms can be obtained by fitting the curve of the stress versus strain. Figure 2 shows the variations of the Young's modulus with the thicknesses of the nanofilms. For the $\mathrm{Ni}(100)$ film which has an outward relaxation, its Young's modulus decreases as the thickness of the film decreases, while the Young's modulus of $\mathrm{Ni}(110)$ and $\mathrm{Ni}(111)$ films increases with the decrease of the film thickness. This characteristic can be regarded as the result of the inward relaxation of the surface. The size-dependent properties of the Young's modulus are also in agreement with the theoretical results qualitatively. ${ }^{3}$ It is expected that, as the thickness is further increased, the Young's moduli eventually approach their respective bulk values. Increasing the strain to make the nanofilms to yield, the yield stress of the films with different thicknesses can be obtained. And the yield stress increases with the decrease of the thickness, which also shows sizedependent characteristics in Fig. 3. This property becomes unobvious when the thickness of the film is thicker than $3.0 \mathrm{~nm}$.

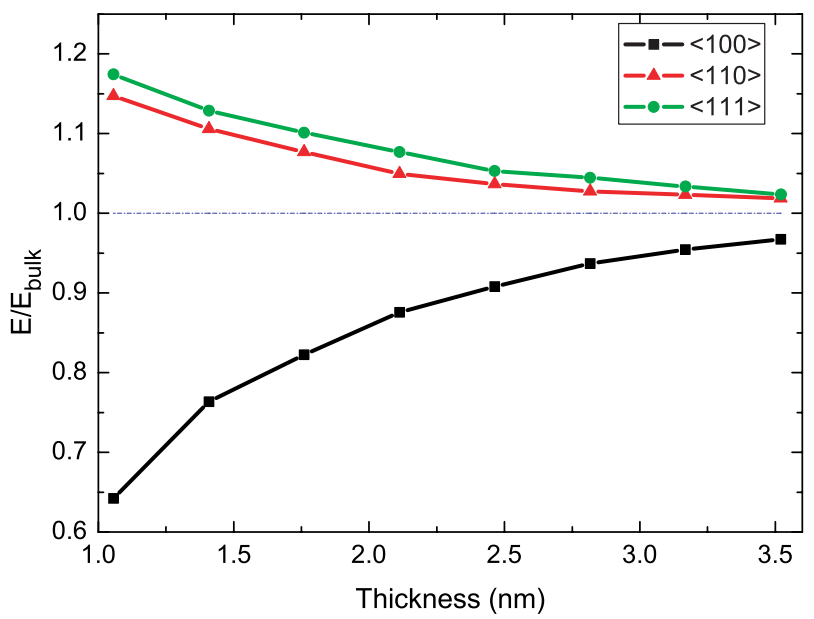

Fig. 2. Young's modulus as a function of thickness of the $\mathrm{Ni}$ nanofilms.

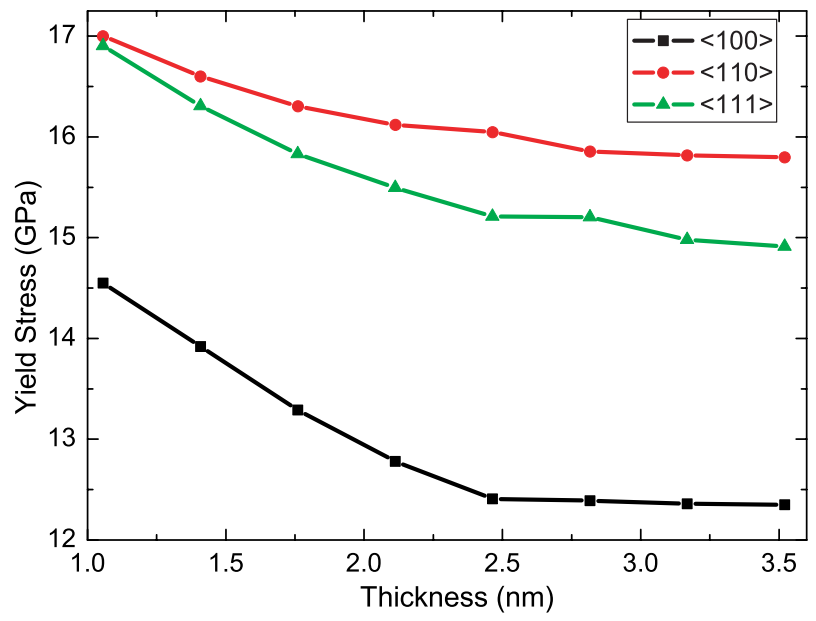

Fig. 3. Yield stress vs thickness of the Ni nanofilms.

\section{Conclusions}

MD simulations of the surface relaxation and the tensile deformation of $\mathrm{Ni}$ nanofilms are carried out to analyze their surface characteristics and elastic properties. The surface relaxations of Ni nanofilms are calculated by tracing the distances between the top five layer atoms. Orientation effects on surface relaxation and surface stress are found. Size dependence is also observed in the elastic responses. The Young's modulus and yield stresses are obtained as functions of thickness and crystallographic orientation. When the surface relaxation is outward, the Young's modulus decreases with the decrease of the film thickness; otherwise, the inward relaxation results in Young's modulus increasing with the decrease of the film thickness. The simulation results also show that the yield stresses of the films increase with the films' thicknesses becoming thinner. With the thickness of the nanofilm decreasing, the surface effects on elastic properties become dominant, which are also expected to occur in other FCC metals.

\section{Acknowledgments}

This work was supported by the National Basic Research Program of China (973 Program, Grant No. 2007CB310504), Distinguished Young Scholar Fund of National Natural Science Foundation of China (NSFC, Grant No. 10225209) and key project from Chinese Academy of Sciences (Grant No. KJCX-SW-L2). 


\section{References}

1. H. G. Graighead, Science 290 (2000) 1532.

2. L. G. Zhou and H. Huang, Appl. Phys. Lett. 84 (2004) 1940.

3. J. G. Guo and Y. P. Zhao, J. Appl. Phys. 98 (2005) 074306 .

4. K. V. Workum and J. J. de Pablo, Phys. Rev. E. 67 (2003) 031601.

5. L. H. Liang, J. C. Li and Q. Jiang, Solid State Commun. 121 (2002) 453.

6. J. K. Diao, K. Gall and M. L. Dunn, Nature Mater. 2 (2003) 656.

7. P. Gumbsch and M. S. Daw, Phys. Rev. B. 44 (1991) 3934.

8. J. Wan, Y. L. Fan, D. W. Gong, S. G. Shen and X. Q. Fan, Modeling Simul. Mater. Sci. Eng. 7 (1999) 189.

9. Y. Kondo, Q. Ru and K. Takayanagi, Phys. Rev. Lett. 82 (1999) 751.
10. S. Cuenot, S. Demoustier-Champagne and B. Nysten, Phys. Rev. Lett. 85 (2000) 1690.

11. V. Chis and B. Hellsing, Phys. Rev. Lett. 93 (2004) 226103.

12. M. S. Daw and M. I. Baskes, Phys. Rev. B 29 (1984) 6443.

13. S. Nosé, J. Chem. Phys. 81 (1984) 511.

14. W. G. Hoover, Phys. Rev. A. 31 (1985) 1695.

15. S. J. Plimpton, J. Comput. Phys. 117 (1995) 1.

16. LAMMPS computer code, http://lammps.sandia. gov/index.html, 2006.

17. R. C. Cammarata, Prog. Surf. Sci. 46 (1994) 1.

18. S. P. Shen and S. N. Atluri, CMES-Comp. Model. Eng. Sci. 6 (2004) 91.

19. J. E. Demuth, P. M. Marcus and D. W. Jepsen, Phys. Rev. B 11 (1975) 1460.

20. S. Hong, A. Kara and T. S. Rahman, Phys. Rev. B. 69 (2004) 195403. 